\title{
Q-space quantitative diffusion MRI measures using a stretched-exponential representation
}

\author{
Tomasz Pieciak ${ }^{1,2[0000-0002-7543-3658]}$, Maryam Afzali3 ${ }^{30000-0003-3378-0878]}$, \\ Fabian Bogusz ${ }^{2}$, Aja-Fernández ${ }^{10000-0002-5337-5071]}$, and Derek K. \\ Jones $3[0000-0003-4409-8049]$ \\ 1 LPI, ETSI Telecomunicación, Universidad de Valladolid, Valladolid, Spain \\ 2 AGH University of Science and Technology, Kraków, Poland \\ 3 Cardiff University Brain Research Imaging Centre (CUBRIC), \\ School of Psychology, Cardiff University, Cardiff, United Kingdom
}

\begin{abstract}
Diffusion magnetic resonance imaging (dMRI) is a relatively modern technique used to study tissue microstructure in a non-invasive way. Non-Gaussian diffusion representation is related to the restricted diffusion and can provide information about the properties of the underlying tissue. In this paper, we analytically derive $n$-th order statistics of the signal considering a stretched-exponential representation of the diffusion and thus retrieve the Q-space quantitative measures such as the Return-To-the-Origin Probability (RTOP), Q-space mean square displacement (QMSD), and Q-space mean fourth-order displacement (QMFD). The stretched-exponential representation enables to handle of the diffusion contributions from a higher $b$-value regime under a non-Gaussian assumption which can be useful in diagnosing or prognosis neurodegenerative diseases in the early stages.
\end{abstract}

Keywords: Diffusion-weighted imaging · diffusion MRI · non-Gaussian diffusion · microstructural measures · Return-To-the-Origin Probability.

\section{Introduction}

Magnetic resonance imaging (MRI) is a powerful technique in clinical applications for diagnoses or prognoses of several diseases in the central nervous system $[8,20]$. Diffusion-weighted MRI (dMRI) modality is sensitive to the random motion of water molecules in the tissue and it is vastly used in both clinical and basic science to characterize tissue water behavior. In the category of dMRI studies, different imaging techniques can extract the microstructural features of the tissue such as size, shape, and anisotropy. A lot of studies have shown a relationship between the changes in the diffusion properties of the tissue and the relevant alteration in the underlying tissue microstructure [29].

One of the most important features of dMRI is its sensitivity to the anisotropy in the tissue. Diffusion tensor imaging (DTI) [8] is the most common technique in clinical studies. The spins displacement in DTI is assumed to be Gaussian distributed and some scalar anisotropy indices such as fractional anisotropy (FA), 
axial, and radial diffusivity (AD, RD), and mean diffusivity (MD) were defined directly from second-order tensor representation $[7,32]$. The most common and simplest assumption in dMRI is the Gaussian assumption [19] on the spin displacement which results in a mono-exponential decay of a diffusion signal versus the $b$-value parameter [11]. The Gaussian assumption in DTI is valid when the media is a simple fluid and we have free diffusion. In more complicated structures such as tissue, there are restrictions in the diffusion of water and therefore the decay is much slower than a mono-exponential decay [9]. The presence of non-mono-exponential decay shows that the diffusion is restricted by the underlying microstructure of the tissue. If the size of the structure is similar to the diffusion length-scale then the diffusion deviates from the Gaussian towards a non-Gaussian displacement regime [16]. Therefore diffusion MRI can be used to probe the microstructural properties of the underlying tissue geometry by methods such as bi-exponential [12], stretched-exponential [9], composite hindered and restricted model of diffusion (CHARMED) [4], AxCaliber [5], ActiveAx [3] or neurite orientation dispersion and density imaging (NODDI) [35]. All these methods can be used to investigate tissue geometry, but they are not all equally applicable in all situations. Other methods such as the high angular resolution diffusion imaging (HARDI) [27,31] and diffusion kurtosis imaging (DKI) [18] were also proposed. To obtain the non-Gaussian property of the signal higher $b$-values are required [21].

Alternative to model the underlying properties of the tissue is the ensemble average propagator (EAP) which represents the probability that the water molecule moves in a specific direction under a certain diffusion time [13, 26]. From the EAP representation one can retrieve different Q-space quantitative measures such as the Return-To-the-Origin Probability (RTOP), Q-space mean square displacement (QMSD), or Q-space mean fourth-order displacement (QMFD). For instance, the RTOP measure is shown to be a good index for cellularity and diffusion restrictions [6] while QMSD and QMFD are sensitive to contributions from slow or restricted diffusion [23].

Different methods have been proposed so far to estimate the EAP and EAPrelated features such as the multiple q-shell diffusion propagator Imaging (mqDPI) [13], Hybrid Diffusion Imaging (HYDI) [34], Mean Apparent Propagator (MAP-MRI) [26], Radial Basis Functions (RBFs) [23] Laplacian-regularized MAP-MRI (MAPL) [14], and Generalized Diffusion Spectrum MRI (GDSI) [30]. These techniques are typically computationally intensive or require a huge amount of densely sampled Cartesian or multiple-shell data to correctly estimate the EAP and its related features. Recently, a single-shell technique that can estimate micro-structure diffusion scalar measures directly from the data has been proposed $[1,28]$. This approach, although enables one to estimate the measures rapidly and directly from the data, assumes a Gaussian profile of the signal, thus it might be problematic to recover higher $b$-value contributions to the signal.

In this paper, we analytically derive the $n$-th order statistics of the signal considering a stretched-exponential decay to represent the Gaussian and nonGaussian part of the signal. In practice, when no information about the number 
of compartments is provided, the stretched exponential is a good choice [10]. Given the general formulation in the Q-space domain, we obtain closed-form formulas to retrieve basic indexes such as the RTOP, QMSD, or QMFD directly from the data in a manner analogous to direct techniques $[1,28,34]$. However, the proposal is no longer limited by a Gaussian assumption and can be used to retrieve the diffusion contributions under the higher $b$-values regime.

\section{Theory}

In this section, we start with the definition of the EAP and diffusion MR signal representation using a stretched-exponential function, and then we use this representation to extract the Q-space scalar measures such as the RTOP, QMSD, and QMFD.

\subsection{Diffusion MR signal representation}

The ensemble average propagator (EAP) is a three-dimensional probability density function that represents the average displacement of spins during the diffusion time. The EAP, $P(\mathbf{R})$, is related to the diffusion MR signal attenuation $E(\mathbf{q})$ via the Fourier transform $[13,26,34]$

$$
P(\mathbf{R})=\int_{\mathbb{R}^{3}} E(\mathbf{q}) \exp \left(-j 2 \pi \mathbf{q}^{T} \mathbf{R}\right) d^{3} \mathbf{q}, \quad j^{2}=-1,
$$

with $E(\mathbf{q})=S(\mathbf{q}) / S(0)$ being the normalized diffusion signal, $S(\mathbf{q})$ is the diffusion signal acquired at wave vector $\mathbf{q}, S(0)$ is the baseline measured without a diffusion sensitization.

The signal in Eq. (1) can be represented by a mono-exponential decay $E(\mathbf{g})=$ $\exp \left(-b \mathbf{g}^{T} \mathbf{D g}\right)$ with $\mathbf{g}$ being a normalized vector $\mathbf{g}=\mathbf{q} /\|\mathbf{q}\|$ and $\mathbf{D}$ is a covariance matrix of a Gaussian EAP or a more general Kohlrausch-Williams-Watts function so-called a stretched-exponential representation given by $[9,22,33]$

$$
E(\mathbf{g})=\exp \left(-(b D(\mathbf{g}))^{\alpha(\mathbf{g})}\right), \quad \alpha(\mathbf{g}) \in(0,1]
$$

with the so-called the $b$-value $b=4 \pi^{2} \tau\|\mathbf{q}\|^{2}\left[\mathrm{~s} / \mathrm{mm}^{2}\right]$ with $\tau=\Delta-\delta / 3[\mathrm{~s}]$ being the effective diffusion time, $D(\mathbf{g})$ and $\alpha(\mathbf{g})$ being the apparent diffusion and stretching parameters at direction $\mathbf{g}$, respectively. Notice here once the stretching parameter tends to unitary, i.e., $\alpha(\mathbf{g}) \rightarrow 1$, the stretched-exponential representation (2) reduces to a mono-exponential signal decay.

\subsection{Q-space domain quantitative measures}

In what follows, we analytically derive $n$-th order statistics of the stretchedexponential representation given by the Eq. (2). This enables to easily retrieve 
three quantitative Q-space measures namely the RTOP $\left[\mathrm{mm}^{-3}\right]$ being the probability in the origin, $P(\mathbf{0})$, QMSD $\left[\mathrm{mm}^{-5}\right]$ and QMFD $\left[\mathrm{mm}^{-7}\right]$ defined as the second- and fourth-order statistics of the signal $E(\mathbf{q})$ respectively $[13,23]$

$\mathrm{RTOP}=\int_{\mathbb{R}^{3}} E(\mathbf{q}) d^{3} \mathbf{q}, \quad \mathrm{QMSD}=\int_{\mathbb{R}^{3}}\|\mathbf{q}\|^{2} E(\mathbf{q}) d^{3} \mathbf{q}, \quad \mathrm{QMFD}=\int_{\mathbb{R}^{3}}\|\mathbf{q}\|^{4} E(\mathbf{q}) d^{3} \mathbf{q}$,

where $\|\cdot\|$ is the vector norm of the wave vector $\mathbf{q}$.

We specify now a more general equation in the Q-space domain related to the $n$-th order statistics of the signal attenuation $E(\mathbf{q})$. Considering the stretchedexponential representation of the signal (2) and a spherical coordinate system $(q, \theta, \varphi)$ with polar $\theta$ and azimutal $\varphi$ angles, and a radial coordinate $q=\|\mathbf{q}\|$ $\left[\mathrm{mm}^{-1}\right]$ we define the $n$-th order statistics of the signal attenuation $E(\mathbf{q})$

$$
\begin{aligned}
M_{n} & =\int_{\mathbb{R}^{3}}\|\mathbf{q}\|^{n} \exp \left(-\left(4 \pi^{2} \tau\|\mathbf{q}\|^{2} D(\mathbf{g})\right)^{\alpha(\mathbf{g})}\right) d^{3} \mathbf{q} \\
& =\int_{0}^{2 \pi} \int_{0}^{\pi} \int_{0}^{\infty} \exp \left(-\left(4 \pi^{2} \tau q^{2} D(\theta, \varphi)\right)^{\alpha(\theta, \varphi)}\right) q^{n+2} \sin \theta d q d \theta d \varphi
\end{aligned}
$$

where $D(\theta, \varphi)$ and $\alpha(\theta, \varphi)$ are the apparent diffusion coefficient and stretching parameter both defined in the spherical coordinate system. Next, we rewrite the integral (4) as follows (see Gradshteyn \& Ryzhik [15], p. 370, Eq. 3.478(1))

$$
\begin{aligned}
M_{n} & =C_{n}^{\tau} \int_{0}^{2 \pi} \int_{0}^{\pi} \Gamma\left(\frac{n+3}{2 \alpha(\theta, \varphi)}\right) \alpha^{-1}(\theta, \varphi) D^{-(n+3) / 2}(\theta, \varphi) \sin \theta d \theta d \varphi \\
& =C_{n}^{\tau} \iint_{\Sigma} \Gamma\left(\frac{n+3}{2 \alpha(\theta, \varphi)}\right) \alpha^{-1}(\theta, \varphi) D^{-(n+3) / 2}(\theta, \varphi) d \Sigma,
\end{aligned}
$$

where $C_{n}^{\tau}=2^{-n-4} \pi^{-n-3} \tau^{-(n+3) / 2}$ is a diffusion time dependent constant and $\Gamma(\cdot)$ is the gamma function. Notice here that the last equation is a surface integral over the surface with a unitary radius, i.e., $q=1$.

\section{$2.3 \quad$ Numerical implementation}

To evaluate the surface integral (5), one can assume the surface area element, $\Delta \Sigma$, is inversely proportional to the number of sampled data points (e.g., the number of evenly distributed directions $N_{g}, \Delta \Sigma=4 \pi / N_{g}$ ). Transforming the Eq. (2) the diffusion becomes $D(\mathbf{g})=4^{-1} \pi^{-2} \tau^{-1} q^{-2}(-\log E(\mathbf{q}))^{1 / \alpha(\mathbf{g})}$, and thus the Eq. (5) can be rewritten in the following form

$$
M_{n}^{(1)}=\frac{1}{2} q^{n+3}\left\langle\Gamma\left(\frac{n+3}{2 \alpha(\mathbf{g})}\right) \alpha^{-1}(\mathbf{g})(-\log E(\mathbf{q}))^{-\frac{n+3}{2 \alpha(\mathbf{g})}}\right\rangle_{\mathbf{q} \in \mathbb{S}^{2}}
$$

with $\langle\cdot\rangle_{\mathbf{q} \in \mathbb{S}^{2}}$ being a direction-averaged signal over a single acquisition shell. 
Notice that the Eq. (6) can be evaluated using the samples retrieved from the resampled data to uniformly cover the surface (e.g., the spherical harmonics basis as in [1]).

The numerical reciprocal of the negative log-diffusion function given in Eq. (6) might be prone to instabilities, i.e., the signal attenuation $E(\mathbf{q}) \rightarrow 1$ the function $(-\log (E(\mathbf{q})))^{-1} \rightarrow \infty$. [2,28]. Alternatively, one can refine (6) to incorporate its second-order series expansion. To this end, we define a twice differentiable function $f: \mathbb{R} \rightarrow \mathbb{R}$ given by $f(X)=\Gamma\left(\frac{n+3}{2 \alpha}\right) \alpha^{-1} X^{-(n+3) /(2 \alpha)}$ with $n \geq 0$. The second-order series expansion of the expectation of the function $f(X)$ around the expectation $\mathbb{E}\{X\}$ is given then by $\mathbb{E}\{f(X)\} \approx f(\mathbb{E}\{X\})+\left.\frac{1}{2} \frac{d^{2} f}{d X^{2}}\right|_{X=\mathbb{E}\{X\}}$. $\operatorname{Var}\{X\}$. After using some algebra we arrive at the following closed-form formula

$$
\begin{gathered}
\mathbb{E}\{f(X)\} \approx \frac{1}{8} \Gamma\left(\frac{n+3}{2 \alpha}\right) \alpha^{-3} \mathbb{E}\{X\}^{-\frac{n+3}{2 \alpha}}\left((n+3)(n+3+2 \alpha) \mathbb{E}\left\{X^{2}\right\} \mathbb{E}\{X\}^{-2}\right. \\
\left.+8 \alpha^{2}-(n+3)(n+3+2 \alpha)\right) .
\end{gathered}
$$

Given again a stretched-exponential decay (2) and a second-order series expansion of the expectation we define an approximation to the measure (6)

$$
\begin{aligned}
& M_{n}^{(2)}= \frac{1}{2} q^{n+3}\left\langle\frac{1}{8} \Gamma\left(\frac{n+3}{2 \alpha(\mathbf{g})}\right) \alpha^{-3}(\mathbf{g})\right\rangle_{\mathbf{q} \in \mathbb{S}^{2}}\langle-\log E(\mathbf{q})\rangle_{\mathbf{q} \in \mathbb{S}^{2}}^{-\left\langle\frac{n+3}{2 \alpha(\mathbf{q})}\right\rangle_{\mathbf{q} \in \mathbb{S}^{2}}} \\
& \times {\left[\frac{(n+3)\langle(n+3) \mathbf{1}+2 \alpha(\mathbf{g})\rangle_{\mathbf{q} \in \mathbb{S}^{2}}\left\langle(-\log E(\mathbf{q}))^{2}\right\rangle_{\mathbf{q} \in \mathbb{S}^{2}}}{\langle-\log E(\mathbf{q})\rangle_{\mathbf{q} \in \mathbb{S}^{2}}}\right.} \\
&+\left\langle 8 \alpha^{2}(\mathbf{g})-2(n+3) \alpha(\mathbf{g})-(n+3)^{2} \mathbf{1}\right\rangle_{\mathbf{q} \in \mathbb{S}^{2}}
\end{aligned}
$$

with 1 being the all-ones vector. We have simplified our derivations in the series expansion procedure; thus, we direction average the stretching parameter to obtain the final formula. From Eq. (7) we can define basic Q-space domain measures such as the RTOP $\left(M_{0}\right)$, QMSD $\left(M_{2}\right)$ or QMFD $\left(M_{4}\right.$; see Eq. (3)). The proposed stretched-exponential method requires a multiple-shell acquisition with at least two-shells at different $b$-values to fit the representation given by Eq. (2). Once the representation is fitted a single-shell data at a fixed $b$-value is used to calculate the measures. In section 2.4, we define a simple optimization cost function to retrieve the stretched representation of the diffusion signal.

\subsection{Optimization of stretched-exponential representation}

To retrieve a stretched-exponential representation at direction $\mathbf{g}$ of the diffusion we define an optimization cost function and solve it using a non-linear least 
squares procedure

$$
(D(\mathbf{g}), \alpha(\mathbf{g}))=\underset{D^{\prime}(\mathbf{g}), \alpha^{\prime}(\mathbf{g})}{\operatorname{argmin}} \frac{1}{2} \sum_{\mathbf{q}: \mathbf{q} \| \mathbf{g}}\left[S(\mathbf{q})-S(0) \exp \left(-\left(4 \pi^{2} \tau\|\mathbf{q}\|^{2} D^{\prime}(\mathbf{g})\right)^{\alpha^{\prime}(\mathbf{g})}\right)\right]^{2} .
$$

To find the optimal parameters we used a bound-constrained minimization via the trust region reflective method with a linear loss function. Notice here the procedure (2.4) applies for each direction $\mathbf{g}$ independently and might use only a subset of $\mathbf{q}$-values employed to acquire the data.

\section{Materials and methods}

In this study, we used ex vivo rat brain data as well as in vivo human brain data that was publicly available by Hansen et al. [17].

\subsection{Ex vivo rat brain data}

The ex vivo data were collected using a Bruker Biospec 9.4T (Bruker Biospin, Germany) with a $15 \mathrm{~mm}$ quadrature coil. Diffusion-weighted images were acquired in $15 b$-value shells ranging from 0 to $5000 \mathrm{~s} / \mathrm{mm}^{2}$ with a step size of 200 $\mathrm{s} / \mathrm{mm}^{2}$ and 33 directions per each shell utilizing a spin echo sequence. Fifteen axial slices were collected at a resolution of $100 \times 100 \times 500 \mu \mathrm{m}^{3}$, matrix size $128 \times 128$, echo time of $\mathrm{TE}=23.3 \mathrm{~ms}$, repetition time of $\mathrm{TR}=4 \mathrm{~s}$, and diffusion timing of $\delta / \Delta=4 / 14 \mathrm{~ms}$. The data set was averaged twice to improve the signal-to-noise ratio being around 75 at the baseline.

\subsection{In vivo Human brain data}

One healthy participant was scanned in an in vivo study using a Siemens Trio $3 \mathrm{~T}$ equipped with a 32 channel head coil. The protocol comprised one $b=0$ and 15 non-zero shells ranging from $200 \mathrm{~s} / \mathrm{mm}^{2}$ to $3000 \mathrm{~s} / \mathrm{mm}^{2}$ with the step size of $200 \mathrm{~s} / \mathrm{mm}^{2}$ and 33 directions per shell. Nineteen axial slices with a voxel size of $2.5 \mathrm{~mm}$ isotropic and a $96 \times 96$ matrix size, $\mathrm{TE}=116 \mathrm{~ms}, \mathrm{TR}=7200$ $\mathrm{ms}$, TI $=2100 \mathrm{~ms}$ were obtained. The diffusion timings were estimated to be $\delta / \Delta=29 / 58 \mathrm{~ms}$. The SNR of the baseline signal is around 39. In our experiments we used a five-shell acquisition with 200, 1000, 18002400 , and $3000 \mathrm{~s} / \mathrm{mm}^{2}$.

\subsection{Comparison to the $\mathrm{Q}$-space measures from different methods}

In subsection 2.2 we introduced three measures that is the RTOP, QMSD and QMFD. In this work, we evaluate the proposed stretched-exponential Q-space measures and compare them to those obtained from the MAP-MRI technique [26] (positivity constraint), MAPL [14] (regularization parameter $\lambda=0.2$ ), $\mathrm{RBF}[23]$ ( $l_{1}$ regularization with $\left.\lambda=0.00055\right)$, 3D-SHORE [25,36] (scale factor 
$\left.\zeta=1 /\left(8 \pi^{2} \tau D\right)\right)$, and a single-shell approach $[1,28]$. Except for the aforementioned frameworks, we calculate also the RTOP measure directly from diffusion tensor eigenvalues (a non-linear least squares fitting via the LevenbergMarquardt method) as RTOP $=(3 \pi \tau)^{-3 / 2}\left(\lambda_{1} \lambda_{2} \lambda_{3}\right)^{-1 / 2}$.

\section{Results and discussion}

In the first experiment, we visually evaluate the measures using ex vivo rat brain data retrieved using different methodologies namely the DTI (at 1000 and $1400 \mathrm{~s} / \mathrm{mm}^{2}$ ), RBF, 3D-SHORE, MAP-MRI, MAPL (the EAPs in all four are fitted to a three-shells acquisition, i.e., $b=1000,3000$ and $5000 \mathrm{~s} / \mathrm{mm}^{2}$ ), single-shell technique, and proposed stretched-exponential one (see Fig. 1). For the proposed technique we use three-shells for fitting the representation while a single-shell to calculate the measure. Visually inspecting the RTOP measure shows that DTI based ones (Fig. 1(a, b)) and single-shell technique (Fig. 1(df)) return smaller values compared to all other methods. The RBF and 3DSHORE have the lowest contrast between the white matter and gray matter tissue in the measure (Fig. 1(c, g)). In the single-shell method by increasing the $b$ value from 1000 to $5000 \mathrm{~s} / \mathrm{mm}^{2}$, the RTOP value increases (Fig. 1(d-f, RTOP)). Comparing the EAP-based techniques and stretched-exponential representations (Fig. 1 (c, g-l, RTOP)), 3D-SHORE and RBF provide the lowest while MAPMRI gives the highest intensity in white matter areas. MAPL results of RTOP

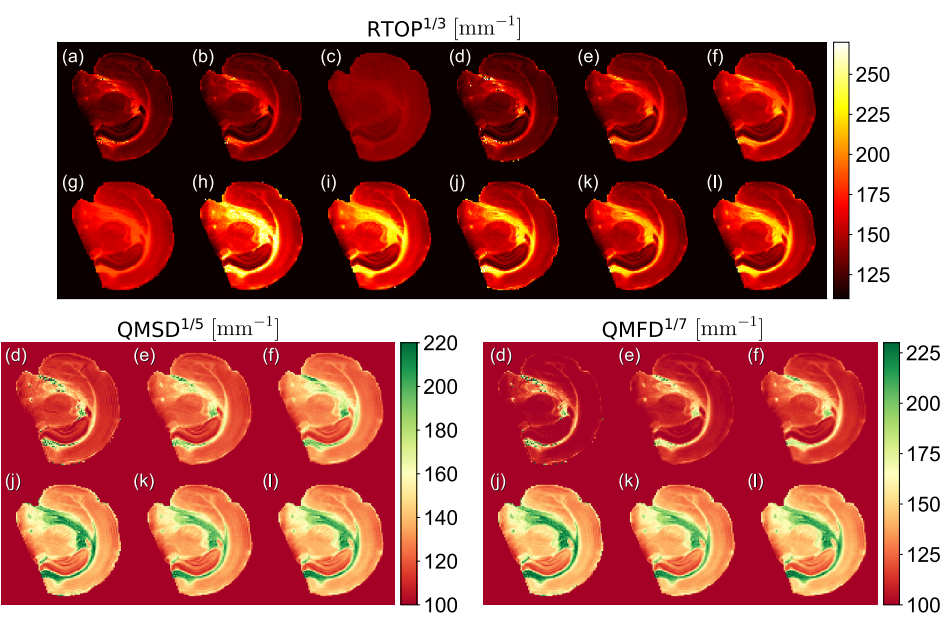

Fig. 1: Visual inspection of the RTOP, QMSD and QMFD measures on ex vivo rat brain data: (a) DTI $\left(b=1000 \mathrm{~s} / \mathrm{mm}^{2}\right)$, (b) DTI $\left(b=1400 \mathrm{~s} / \mathrm{mm}^{2}\right)$, (c) RBF, (d) single-shell $\left(b=1000 \mathrm{~s} / \mathrm{mm}^{2}\right)$, (e) single-shell $\left(b=3000 \mathrm{~s} / \mathrm{mm}^{2}\right)$, (f) singleshell $\left(b=5000 \mathrm{~s} / \mathrm{mm}^{2}\right)$, (g) 3D-SHORE, (h) MAP-MRI, (i) MAPL, (j) stretchedexponential $\left(b=1000 \mathrm{~s} / \mathrm{mm}^{2}\right),(\mathrm{k})$ stretched-exponential $\left(b=3000 \mathrm{~s} / \mathrm{mm}^{2}\right)$ and (l) stretched-exponential $\left(b=5000 \mathrm{~s} / \mathrm{mm}^{2}\right)$. 


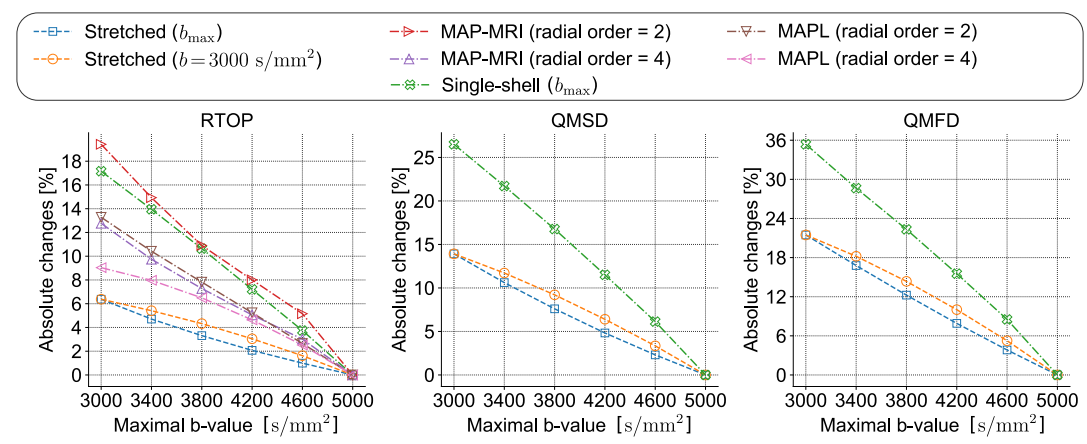

Fig. 2: The mean absolute changes of the RTOP, QMSD and QMFD measures in ex vivo rat brain data in terms of maximal $b$-value $\left(b_{\max }\right)$ used to estimate the EAP/calculate the measure under different techniques. For stretchedexponential representation two variants are used in measure calculation process namely $b$-value at $b_{\max }$ and $b=3000 \mathrm{~s} / \mathrm{mm}^{2}$.
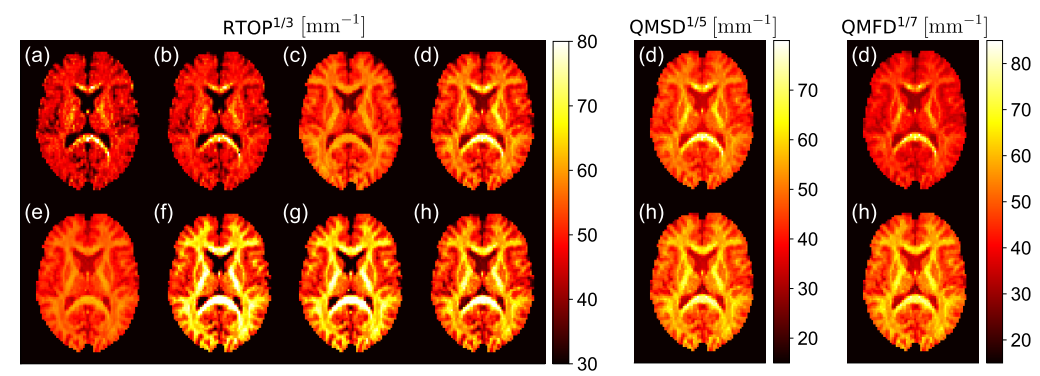

Fig. 3: Visual inspection of the measures on in vivo human brain data estimated using various approaches: (a) DTI $\left(b=1000 \mathrm{~s} / \mathrm{mm}^{2}\right)$, (b) DTI $\left(b=1400 \mathrm{~s} / \mathrm{mm}^{2}\right)$, (c) RBF, (d) single-shell $\left(b=3000 \mathrm{~s} / \mathrm{mm}^{2}\right)$, (e) 3D-SHORE, (f) MAP-MRI, (g) MAPL and (h) stretched-exponential $\left(b=3000 \mathrm{~s} / \mathrm{mm}^{2}\right)$.

are similar to MAP-MRI while the RTOP values in MAPL are slightly lower than the MAP-MRI. Our proposed method of stretched-exponential provides similar RTOP maps for different $b$-values $\left(b=1000,3000\right.$, and $\left.5000 \mathrm{~s} / \mathrm{mm}^{2}\right)$ and it preserves the consistency of the measures between different $b$-values which is not observed in single-shell technique (Fig. 1(d-f, j-l, RTOP)). Clearly, introducing the stretched-exponential representation enabled to improve contrast and kept the uniformity of the RTOP measure across the $b$-values. In the QMSD/QMFD measures, single-shell method at $b=1000 \mathrm{~s} / \mathrm{mm}^{2}$ has the lowest value in the both gray matter and white matter while the $b=5000 \mathrm{~s} / \mathrm{mm}^{2}$ has the highest and $b=3000 \mathrm{~s} / \mathrm{mm}^{2}$ is the intermediate between the three alternatives of the single-shell method (Fig. 1 (d-f, QMSD/QMFD). Notice here that again the behaviour of the QMSD/QMFD measures across the $b$-values are preserved and 

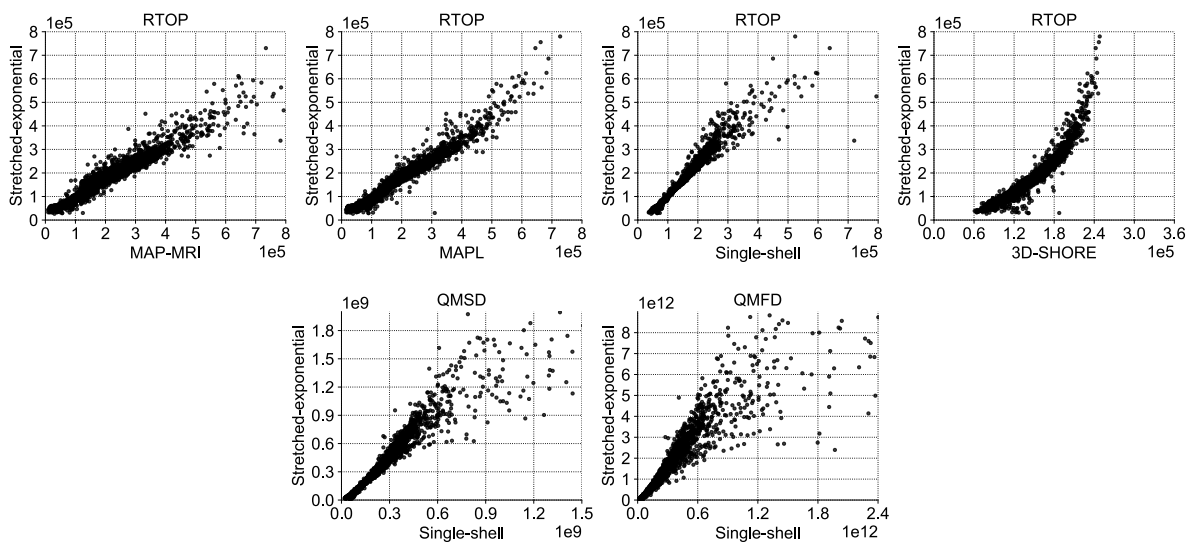

Fig. 4: Correlations between the RTOP retrieved from in vivo human brain data using the proposed stretched-exponential and state-of-the-art methods namely MAP-MRI, MAPL, single-shell and 3D-SHORE (top), and correlations between the proposal and single-shell approach in case of QMSD/QMFD (bottom).

the proposed stretched-exponential representation keeps the consistency of the quantities while changing the $b$-value used to calculate the measure.

In the second experiment, we extrapolate the previous one and evaluate the absolute changes in the measures due to the changes in the maximal $b$-value parameter. In this experiment we used six different acquisitions from threeshells (i.e., $b=1000,2000$ and $b=3000 \mathrm{~s} / \mathrm{mm}^{2}$ ) to eight-shell one (up to $b=5000 \mathrm{~s} / \mathrm{mm}^{2}$ with a step of $400 \mathrm{~s} / \mathrm{mm}^{2}$ ). For the proposed methodology we fit the representation using $k$ shells $(k=3, \ldots, 8$; similarly to the EAPbased methods) while calculate the measures using only one shell namely $b=$ $3000 \mathrm{~s} / \mathrm{mm}^{2}$ and $b_{\max }$. Fig. 2 depicts the mean absolute changes of the RTOP, QMSD, and QMFD measures in terms of maximal $b$-value $\left(b_{\max }\right)$ under different methodologies used to estimate the measures. For the estimation of RTOP, our proposed stretched-exponential methodology has the minimum mean absolute changes for both $b_{\max }$ and $b=3000 \mathrm{~s} / \mathrm{mm}^{2}$ used to retrieve the measure once fitted the Eq. (2.4). It is worth noticing here that the single-shell technique is heavy load due to the changes in the maximal $b$-value and our proposal improved the results though the measure is still calculated from a single-shell. As for the two other measures (QMSD and QMFD), our proposed method is superior to the single-shell method while again the one with $b_{\max }$ is slightly better than that with $b=3000 \mathrm{~s} / \mathrm{mm}^{2}$.

Fig. 3 is devoted to visual inspection of the RTOP and QMSD/QMFD measures on in vivo human brain data estimated using various state-of-the-art approaches. The observed trend in the in vivo maps of RTOP is similar to the one observed in ex vivo data (Fig. 1; RTOP). Again, a comparable behaviour of the QMSD/QMFD measures can be observed, i.e., the single-shell technique exhibit generally smaller values of the measure in white matter areas compared 


\begin{tabular}{l|c|c|c|c|c|c|c|c}
\hline \hline & DTI & MAP-MRI & MAPL & Single (1) & Single (2) & 3D-SHORE & SE (1) & SE (2) \\
\hline DTI & $\times$ & & & & & & & \\
\hline MAP-MRI & 0.681 & $\times$ & & & & & & \\
\hline MAPL & 0.674 & 0.981 & $\times$ & & & & & \\
\hline Single (1) & 0.827 & 0.901 & 0.895 & $\times$ & & & & \\
\hline Single (2) & 0.797 & 0.936 & 0.932 & 0.978 & $\times$ & & & \\
\hline 3D-SHORE & 0.642 & 0.928 & 0.961 & 0.863 & 0.895 & $\times$ & & \\
\hline SE (1) & 0.739 & 0.951 & 0.965 & 0.944 & 0.954 & 0.931 & $\times$ & \\
\hline SE (2) & 0.728 & 0.957 & 0.973 & 0.925 & 0.959 & 0.933 & 0.982 & $\times$ \\
\hline \hline
\end{tabular}

Table 1: Pearson's correlation coefficient between different methodologies used to retrieve the RTOP measure from human brain data (see Fig. 3). Legend: Single (1), (2) - single-shell technique at $b=2400$ and $b=3000 \mathrm{~s} / \mathrm{mm}^{2} ; \mathrm{SE}(1),(2)-$ stretched-exponential at $b=2400$ and $b=3000 \mathrm{~s} / \mathrm{mm}^{2}$, respectively.

to stretched-exponential representation. Notice here that the QMFD measure obtained from the single-shell technique is no longer able to catch heavy tails of the signal distribution properly from the data.

Lastly, we extrapolate the previous experiment using in vivo human data and evaluate the correlation between measures both visually and numerically. Here, we again use a five-shells acquisition to calculate the measures. Fig. 4(top row) illustrates correlations between the RTOP retrieved using the proposed approach and those obtained under different state-of-the-art methods namely MAP-MRI, MAPL, the single-shell approach, and 3D-SHORE over the image mask (white matter, gray matter, and cerebrospinal fluid areas). Our method shows a good correlation with all the other four methods being characterized by Pearson's correlation coefficient equal to $\rho=0.925$ is the worst case (see Table 1). Fig. 4 also shows the correlation between our method and single-shell approach in the case of QMSD and QMFD measures. Notice that the correlograms for QMSD/QMFD measures clearly show the outliers generated by the single-shell technique which are not present in the proposed one. Finally, notice that in situations where the signal cannot be accurately described by the stretchedexponential [24], the scalar measures will be biased.

\section{Conclusions}

In this paper, we proposed a new approach based on stretched-exponentials to quantify EAP features such as RTOP, QMSD, and QMFD measures. From the results, it seems that the proposed method reduces the amount of data to be acquired and therefore it can be clinically feasible. We have to mention that much more thorough validation and comparison are required to bring convincing evidence that comparable results can be obtained with fewer data in our method. Besides, the proposed method can be generalized to other Q-space factors such as return-to-the-axis or return-to-the-plane probability. 


\section{References}

1. Aja-Fernández, S., de Luis-García, R., Afzali, M., Molendowska, M., Pieciak, T., Tristán-Vega, A.: Micro-structure diffusion scalar measures from reduced MRI acquisitions. PLOS ONE 15(3), e0229526 (2020)

2. Aja-Fernández, S., Pieciak, T., Tristán-Vega, A., Vegas-Sánchez-Ferrero, G., Molina, V., de Luis-García, R.: Scalar diffusion-MRI measures invariant to acquisition parameters: a first step towards imaging biomarkers. Magn. Reson. Imaging 54, 194-213 (2018)

3. Alexander, D.C.: A general framework for experiment design in diffusion MRI and its application in measuring direct tissue-microstructure features. Magn. Reson. Med. 60(2), 439-448 (2008)

4. Assaf, Y., Basser, P.J.: Composite hindered and restricted model of diffusion (CHARMED) MR imaging of the human brain. Neuroimage 27(1), 48-58 (2005)

5. Assaf, Y., Blumenfeld-Katzir, T., Yovel, Y., Basser, P.J.: AxCaliber: a method for measuring axon diameter distribution from diffusion MRI. Magn. Reson. Med. 59(6), 1347-1354 (2008)

6. Avram, A.V., Sarlls, J.E., Barnett, A.S., Özarslan, E., Thomas, C., Irfanoglu, M.O., Hutchinson, E., Pierpaoli, C., Basser, P.J.: Clinical feasibility of using mean apparent propagator (MAP) MRI to characterize brain tissue microstructure. NeuroImage 127, 422-434 (2016)

7. Basser, P.J.: Inferring microstructural features and the physiological state of tissues from diffusion-weighted images. NMR Biomed. 8(7), 333-344 (1995)

8. Basser, P., Pierpaoli, C.: Microstructural features measured using diffusion tensor imaging. J. Magn. Reson. B 111(3), 209-219 (1996)

9. Bennett, K.M., Schmainda, K.M., Bennett, R., Rowe, D.B., Lu, H., Hyde, J.S.: Characterization of continuously distributed cortical water diffusion rates with a stretched-exponential model. Magn. Reson. Med. 50(4), 727-734 (2003)

10. Bennett, K., Schmainda, K., Rowe, D., Rebro, K., Hyde, J.: A stretchedexponential model of distributed diffusion rates in brain. In: Proc. ISMRM, 11th Scientific Meeting, Toronto, Canada (2003)

11. Callaghan, P.T.: Translational dynamics and magnetic resonance: principles of pulsed gradient spin echo NMR. Oxford University Press (2011)

12. Clark, C.A., Le Bihan, D.: Water diffusion compartmentation and anisotropy at high b values in the human brain. Magn. Reson. Med. 44(6), 852-859 (2000)

13. Descoteaux, M., Deriche, R., Le Bihan, D., Mangin, J.F., Poupon, C.: Multiple q-shell diffusion propagator imaging. Med. Image Anal. 15(4), 603-621 (2011)

14. Fick, R.H., Wassermann, D., Caruyer, E., Deriche, R.: MAPL: Tissue microstructure estimation using Laplacian-regularized MAP-MRI and its application to HCP data. Neuroimage 134, 365-385 (2016)

15. Gradshteyn, I.S., Ryzhik, I.M.: Table of integrals, series, and products. Academic press (2014)

16. Grebenkov, D.S.: Laplacian eigenfunctions in nmr. i. a numerical tool. Concepts in Magnetic Resonance Part A: An Educational Journal 32(4), 277-301 (2008)

17. Hansen, B., Jespersen, S.N.: Data for evaluation of fast kurtosis strategies, b-value optimization and exploration of diffusion MRI contrast. Sci. data 3(1), 1-5 (2016)

18. Jensen, J.H., Helpern, J.A., Ramani, A., Lu, H., Kaczynski, K.: Diffusional kurtosis imaging: the quantification of non-gaussian water diffusion by means of magnetic resonance imaging. Magn Reson Med 53(6), 1432-1440 (2005) 
19. Jones, D.K., Knösche, T.R., Turner, R.: White matter integrity, fiber count, and other fallacies: the do's and don'ts of diffusion MRI. Neuroimage 73, 239-254 (2013)

20. Kubicki, M., McCarley, R., Westin, C.F., Park, H.J., Maier, S., Kikinis, R., Jolesz, F.A., Shenton, M.E.: A review of diffusion tensor imaging studies in schizophrenia. J. Psychiatr. Res. 41(1-2), 15-30 (2007)

21. Le Bihan, D.: Molecular diffusion nuclear magnetic resonance imaging. Magn. Reson. Q. 7(1), 1 (1991)

22. Magin, R.L., Karani, H., Wang, S., Liang, Y.: Fractional order complexity model of the diffusion signal decay in MRI. Mathematics 7(4), 348 (2019)

23. Ning, L., Westin, C.F., Rathi, Y.: Estimating diffusion propagator and its moments using directional radial basis functions. IEEE Trans. Med. Imaging 34(10), 20582078 (2015)

24. Novikov, D.S., Fieremans, E., Jespersen, S.N., Kiselev, V.G.: Quantifying brain microstructure with diffusion MRI: Theory and parameter estimation. NMR Biomed. 32(4), e3998 (2019)

25. Ozarslan, E., Koay, C., Shepherd, T., Blackb, S., Basser, P.: Simple harmonic oscillator based reconstruction and estimation for three-dimensional q-space MRI (2009)

26. Özarslan, E., Koay, C.G., Shepherd, T.M., Komlosh, M.E., İrfanoğlu, M.O., Pierpaoli, C., Basser, P.J.: Mean apparent propagator (MAP) MRI: a novel diffusion imaging method for mapping tissue microstructure. Neuroimage 78, 16-32 (2013)

27. Özarslan, E., Shepherd, T.M., Vemuri, B.C., Blackband, S.J., Mareci, T.H.: Resolution of complex tissue microarchitecture using the diffusion orientation transform (DOT). Neuroimage 31(3), 1086-1103 (2006)

28. Pieciak, T., Bogusz, F., Tristán-Vega, A., de Luis-García, R., Aja-Fernández, S.: Single-shell return-to-the-origin probability diffusion MRI measure under a nonstationary Rician distributed noise. In: IEEE 16th International Symposium on Biomedical Imaging. pp. 131-134. IEEE (2019)

29. Rovaris, M., Filippi, M.: Diffusion tensor MRI in multiple sclerosis. J. Neuroimaging 17, 27S-30S (2007)

30. Tian, Q., Yang, G., Leuze, C., Rokem, A., Edlow, B.L., McNab, J.A.: Generalized diffusion spectrum magnetic resonance imaging (GDSI) for model-free reconstruction of the ensemble average propagator. Neuroimage 189, 497-515 (2019)

31. Tuch, D.S., Reese, T.G., Wiegell, M.R., Wedeen, V.J.: Diffusion MRI of complex neural architecture. Neuron 40(5), 885-895 (2003)

32. Westin, C.F., Maier, S.E., Mamata, H., Nabavi, A., Jolesz, F.A., Kikinis, R.: Processing and visualization for diffusion tensor MRI. Med. Imag. Anal. 6(2), 93-108 (2002)

33. Williams, G., Watts, D.C.: Non-symmetrical dielectric relaxation behaviour arising from a simple empirical decay function. Transactions of the Faraday society 66 , 80-85 (1970)

34. Wu, Y.C., Field, A.S., Alexander, A.L.: Computation of diffusion function measures in $q$-space using magnetic resonance hybrid diffusion imaging. IEEE Trans. Med. Imaging. 27(6), 858-865 (2008)

35. Zhang, H., Schneider, T., Wheeler-Kingshott, C.A., Alexander, D.C.: NODDI: Practical in vivo neurite orientation dispersion and density imaging of the human brain. Neuroimage 61(4), 1000-1016 (2012)

36. Zucchelli, M., Brusini, L., Méndez, C.A., Daducci, A., Granziera, C., Menegaz, G.: What lies beneath? Diffusion EAP-based study of brain tissue microstructure. Med. Imag Anal. 32, 145-156 (2016) 